\title{
Many-body diffusion algorithm: Harmonic fermions
}

\author{
F. Luczak, F. Brosens, and J. T. Devreese* \\ Departement Natuurkunde, Universiteit Antwerpen (UIA), Universiteitsplein 1, B-2610 Antwerpen, Belgium \\ L. F. Lemmens \\ Departement Natuurkunde, Universiteit Antwerpen (RUCA), Groenenborgerlaan 171, B-2020 Antwerpen, Belgium
}

(Received 16 September 1997)

\begin{abstract}
A numerical implementation scheme is presented for the recently developed many-body diffusion approach for identical particles, in the case of harmonic potentials. The procedure is free of the sign problem, by the introduction of the appropriate absorption or reflection conditions for the walkers at the boundary of a state space. These conditions are imposed by the permutation symmetry. The outflow of the walkers at the boundary of the state space contributes substantially to the energy. Furthermore, the implementation of crossingrecrossing effects at absorbing boundaries proves indispensable to sample the antisymmetric states by discrete time steps. [S1063-651X(98)02802-5]
\end{abstract}

PACS number(s): 02.70.Lq, 05.30.Fk, 03.65.Ca, 02.50.Ga

\section{INTRODUCTION}

In this paper the theoretical method put forward in a series of publications by three of the present authors [1-3] around the criticism [4] on an approach initiated by Korzeniowski et al. [5] is applied to an analytically exact soluble model. A sign-problem-free estimation of an excited antisymmetric eigenstate of the model is presented.

Monte Carlo simulations of an excited state of quantum systems are only possible if symmetry considerations can be invoked to guarantee that the excited state is orthogonal to all lower lying states of the same system. Usually this means that one simulates the state with the lowest energy that transforms according to a particular irreducible representation of the symmetry group of the system. Usually this also means that the state has a nodal structure in the configuration space. This is, e.g., the case if one wants to simulate a system containing a fixed number of fermions. The (unknown) nodal structure of this system is the origin of the so-called sign problem. The fermion state to be simulated is an excited state of a quantum system of distinguishable particles (the walkers in the simulation are registered) that transforms according to the antisymmetric irreducible representation of the permutation group $S_{N}$, where $N$ is the number of indistinguishable particles. Up till now, there is no generally accepted way that solves the sign problem. Several approaches avoid the problem approximately by fixing the nodal surfaces to be those of a known, usually exactly solvable problem, or by allowing that parts of the configuration space with different sign are sampled in combination with variance reducing methods [68].

Oscillators belong to the class of simple models that can be tested easily [9-11] and gain importance in view of the models for trapped systems [12]. The ground-state energy and the static response functions of particles in a harmonic potential can be calculated with their statistics taken into

\footnotetext{
*Also at the Universiteit Antwerpen (RUCA) and Technische Universiteit Eindhoven, The Netherlands.
}

account, even in the presence of harmonic two-body interactions. It is clear that analytic path-integral methods [13-15] allow the study of these models in a more direct way. But it is not without value in view of the sign problem if the lowest energy state of this harmonic system with a specified symmetry can be simulated with a sign-problem-free procedure. Such a procedure will be applied in the present paper. We want to stress that our only inference is that we simulate the model (fermions interacting harmonically and isotropically) sign-problem-free. The adaptation of the technique for a realistic model is beyond the scope of the present paper. The limitation of the proposed method originates from the fact that in the theoretical framework [1-3] leading to this algorithm, we rely on a typical symmetry property of harmonic interactions, which is absent for more general potentials [16]. Nevertheless, the basic idea of using the permutation symmetry to partition the configuration space in domains with boundary conditions offers the potentiality to lead to a general procedure for more realistic systems.

From our theoretical contributions to this problem [1-3], it should be clear that, in an algorithmic way, we have to study a new problem, formerly not encountered in the area of quantum Monte Carlo simulations and relating the sign problem to absorption and reflection of walkers at a boundary. The novelty is that the boundary for a walker depends on the positions of some other walkers.

The introduction of a domain also has consequences for the estimation of the ground-state energy. With ground-state energy we mean here the lowest energy of all the states that transform according to a specified irreducible representation of a symmetry group. The absorption of walkers at the boundary of the domain creates a flux of walkers out of the phase space volume that gives an important contribution to the estimate for the energy as will be shown below.

This paper is organized as follows. In Sec. II we will discuss what absorption or reflection means for the distribution of particles in a domain $D_{N}^{3}$ and how this distribution can be simulated by Bernoulli walks or by diffusion Monte Carlo. In this respect a crossing-recrossing correction deserves special attention. In Sec. III the energy estimation is 
considered, with special attention to the flux term coming from the absorption of walkers at the boundary. In the last section the results for a set of oscillator models are given, followed by a discussion and conclusions.

\section{FREE DIFFUSION WITH A BOUNDARY}

For $N$ particles moving in a $3 N$-dimensional configuration space, permutation symmetry can be taken into account by ordering linearly the coordinates of the particles along the three basic directions, introducing in this way a domain $D_{N}^{3}$. Depending on the configuration the antisymmetric representation can be realized by several combinations of boundary conditions: the antisymmetric representation is irreducible with respect to the permutation of $N$ particles but is reducible with respect to the permutation of the $3 N$ coordinates. The symmetry of the model without interaction or with only harmonic interactions along the principal axes is higher than that of a many-body system with realistic one-body and twobody potentials. A decomposition into irreducible representations of the permutation of coordinates leads to four combinations, labeled by a quantum number $\ell=0,1,2,3$ referring to the behavior of the coordinates at the boundary $\partial D_{N}^{u}, u$ $=x, y, z$. We follow here the conventions introduced in [3]. The case $\ell=0$ for the antisymmetric combination, e.g., means that coordinates behave fermionlike in the three directions, while $\ell=2$, for instance, means that the coordinates behave fermionlike in the $y$ direction and bosonlike in the two other directions. Using this reduction by symmetry and the appropriate boundary conditions, the representative domain that is specified cannot be left by the walkers anymore. On this domain the wave function of the model does not change sign, leading to a sign-problem-free simulation of that antisymmetric state. It is important first to analyze the algorithm for moves in one direction only. This is done for fermionlike as well as for bosonlike behavior. The moves in a general three-dimensional (3D) direction are combinations of both.

\section{A. Reflection and bosons on a line}

For $N$ bosons moving on a line, the domain $D_{N}$, that we consider here, is linearly ordered by $x_{i}>x_{i+1}$ and the boundary of the domain $\partial D_{N}$ is reached if one of the following equations $x_{i}=x_{i+1}$ is satisfied for $i=2, \ldots, N$. A sample path for $N$ walkers starting in the domain is denoted by $X(\tau)$ and the increments of this $N$-dimensional random walker or Brownian motion are independent and obtained as usual. This sample path can be used to construct one with reflection at the boundary $\partial D_{N}$ by the following procedure. Consider the path of one component $i$ with a reflecting boundary at the origin. In this case those parts of $X_{i}(\tau)$ which are outside the domain before the time $\tau$ are reflected to the inside by adding an amount $L_{i}(\tau)$, determined by the most negative value on the trajectory in the time interval $[0, \tau]$,

$$
L_{i}(\tau)=-\inf \left\{X_{i}\left(\tau^{\prime}\right), 0 ; \tau^{\prime} \leqslant \tau\right\} .
$$

The sample path of the diffusion or random walk with reflection is given by the Skohorod construction:

$$
\hat{X}_{i}(\tau)=X_{i}(\tau)+L_{i}(\tau)
$$

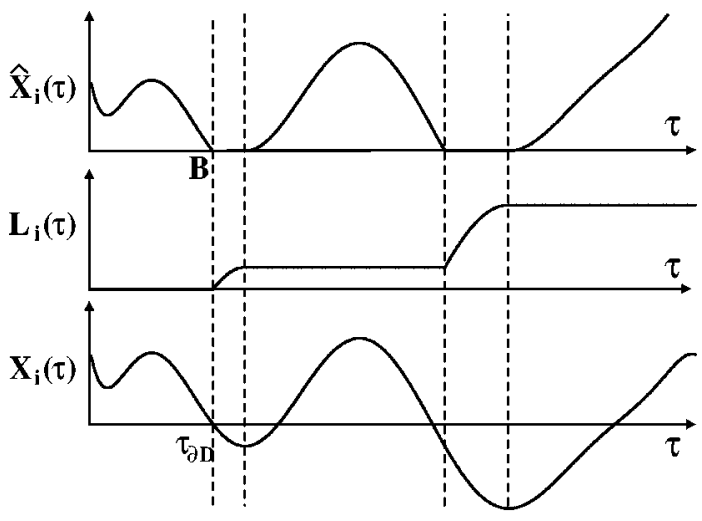

FIG. 1. Illustration of the Skohorod construction for a Brownian trajectory $X_{i}(\tau) . \hat{X}_{i}(\tau)$ refers to the bosonlike random process in the presence of a reflecting boundary ( $\tau$ axis). The corresponding fermionlike process $\widetilde{X}_{i}(\tau)$ is identical to $X_{i}(\tau)$ until the point $B$, whereupon it stays on the boundary propagating with zero weight.

By definition, $L_{i}(\tau)$ is a nondecreasing random function on the event space, and it increases only when $\hat{X}_{i}(t)$ reaches the boundary, i.e., in this example if $\hat{X}_{i}(\tau)=0$. This mechanism is illustrated in Fig. 1, in which the reflection of a Brownian sample path $X_{i}(\tau)$ occurs by the combination of $X_{i}(\tau)$ with $L_{i}(\tau)$. (For vividness but without consequences for the principle in mind, the sample path has been depicted as a smooth function.) With the $N$-dimensional generalization of the construction, it can be shown that the transition probability satisfies the Neumann boundary condition for the backward equation. It should be noted that if a diffusion with drift is considered-the forward and backward equations are different-the boundary conditions will become dependent on the drift [17].

In order to see how the distribution arises in a simulation using prescription (2) for boson diffusion, the example of two identical bosons on a line is instructive. A representative outcome of the numerically simulated free-particle boson diffusion is compared in Fig. 2 with the rigorous density, i.e., a permanent with the appropriate transition probabilities $\rho\left(x_{i}^{\prime}, x_{j} ; \Delta \tau\right)$ as entries, where $x_{i}^{\prime}$ and $x_{j}$ (with $i, j$ $=1, \ldots, N)$ are coordinates at time $\tau$ and at time $\tau+\Delta \tau$,
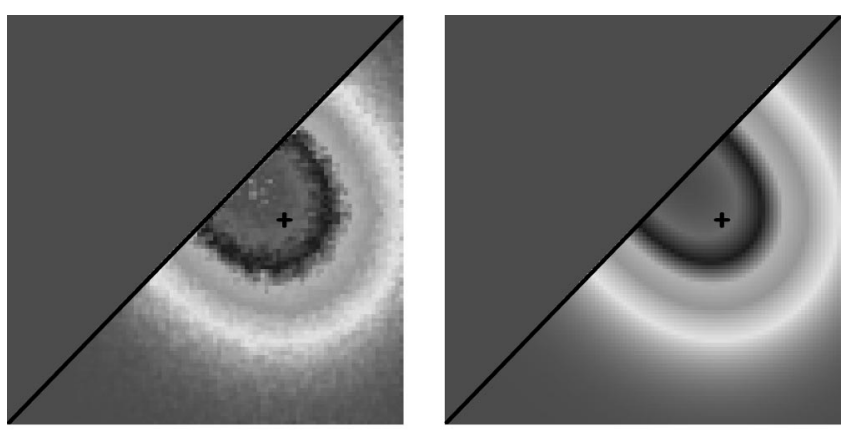

FIG. 2. Comparison of the rigorous (right hand side) and numerically sampled (left-hand side) probability densities of two onedimensional free-diffusive identical bosons, having started from an initial point denoted by the crosses, after an evolution of three atomic time units. The solid line indicates the state space boundary $x_{1}=x_{2}$. 

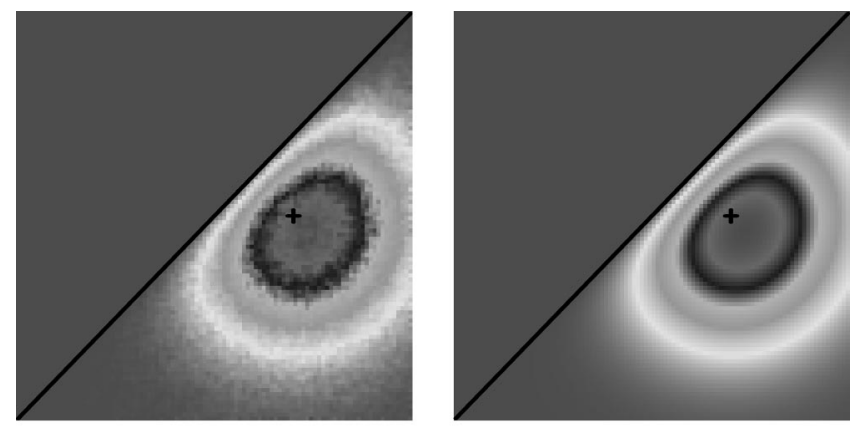

FIG. 3. The same as Fig. 2 for fermions.

respectively. First, an ensemble of walkers was created at a fixed position inside the state space $D_{2}$. The initial position is indicated by a cross, whereas the state space $D_{2}$ with $x_{1}$ $>x_{2}$ is situated right below the solid black lines $x_{1}=x_{2}$. The walkers evolved for three atomic times and by projection of the walker coordinates onto the $\left(x_{1}, x_{2}\right)$ plane a snapshot of the simulated density has been obtained. The numerical density is in good agreement with the rigorous one, also shown in the figure.

\section{B. Absorption and fermions on a line}

For fermions on a line on the same domain $D_{N}$ but with absorbing boundary conditions, the sampling technique has to generate a determinant with, as elements, the $\rho\left(x_{i}^{\prime}, x_{j} ; \Delta \tau\right)$. The construction of a sample path with absorption from a Brownian motion or a random walk $X(\tau)$ proceeds with the introduction of a first-passage time $\tau_{\partial D}$ :

$$
\tau_{\partial D}=\inf \left\{\tau \geqslant 0 ; X(\tau) \in \partial D_{n}\right\}
$$

The new stochastic process $\widetilde{X}(\tau)$ is then by definition

$$
\widetilde{X}(\tau)=\left\{\begin{array}{lll}
X(\tau) & \text { if } & \tau<\tau_{\partial D} \\
X_{\tau_{\partial D}} & \text { if } & \tau \geqslant \tau_{\partial D} .
\end{array}\right.
$$

It should be noted that each sample path $X(\tau)$ has its own first-passage time; therefore this time is a random variable. The transition probability for the process $\widetilde{X}(\tau)$ satisfies the Dirichlet boundary conditions. As argued in [1], this procedure simulates the Slater determinant describing motion on a line.

In order to see how the distribution arises using prescription (4) for fermion diffusion, the simulation of two identical fermions on a line is shown in Fig. 3. Also in this case, the numerical density has been found to be in good agreement with the analytically rigorous one.

\section{Bosons and fermions in one dimension}

Figures 2 and 3 vividly recover typical characteristics attributing to the time evolution of free bosons and fermions. As has been shown in a previous paper [2], the state-space boundary $\partial D_{2}$ acts attractive for the two bosons. Their modal trajectory, i.e., the trajectory with the highest density, rapidly approaches the boundary and stays there for the rest of the evolution time [2]. Fermion evolution on the contrary looks as if the two fermions would be repelled by the bound-
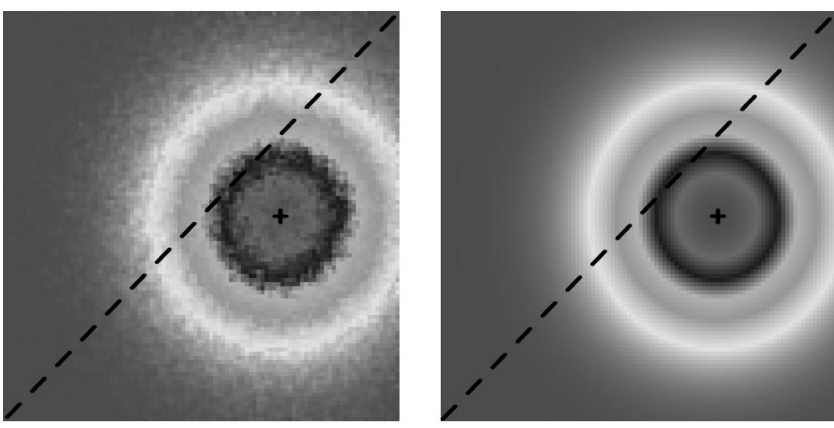

FIG. 4. The same as Fig. 2 for distinguishable particles. The dashed line indicates the hypersurface $x_{1}=x_{2}$.

ary; their modal distance monotonously increases with Euclidean time. These properties are in contrast to the evolution of distinguishable particles, which is not restricted to the state space $D_{2}$ (see Fig. 4). The walkers spread out isotropically with rigid modal distance, which nicely illustrates the lack of free-particle interactions due to quantum statistics.

\section{Absorption and reflection in more than one dimension}

In order to illustrate the construction of an antisymmetric state for two particles using the appropriate processes for diffusion of their coordinates, the rate that the walkers get absorbed at the boundary of the state space is a characteristic of the many-body diffusion algorithm. In three spatial dimensions and for an antisymmetric state, the four fermion processes $\{f, b, b\},\{b, f, b\},\{b, b, f\}$, and $\{f, f, f\}$ introduced in [3] generally reveal different absorption rates. Figure 5 gives a typical example for the (Euclidean) time dependence of the relative number of walkers residing in the state space. Providing infinitely large samples, this numerical quantity converges to the conditional probability $P_{\bar{x}}\left(\tau_{\partial D}>\tau\right)$. Due to the presence of absorbing boundaries in each direction, the $\{f, f, f\}$ process decays faster than any of the other three

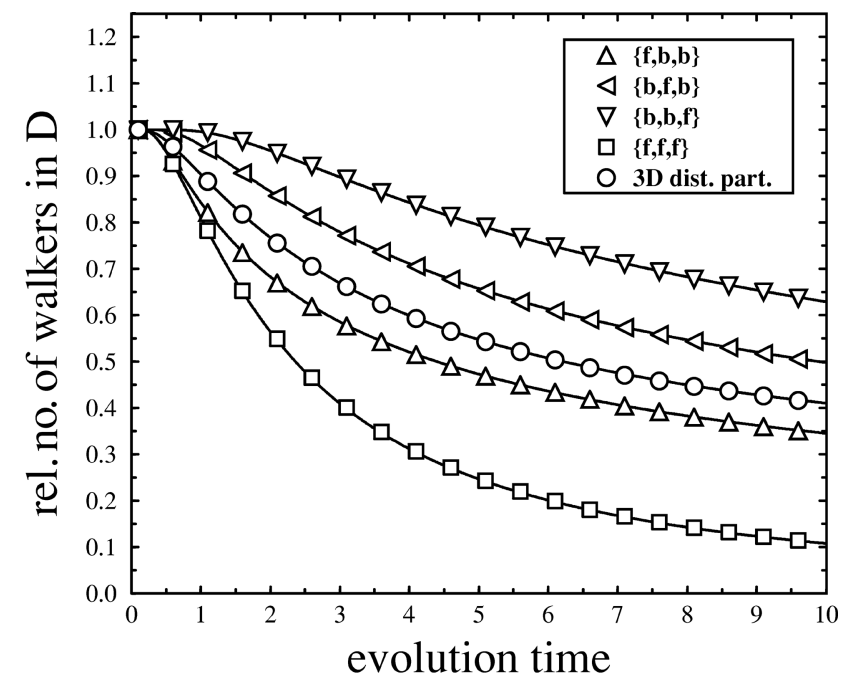

FIG. 5. An exemplary comparison of the numerically obtained relative number of walkers inside the state space with the rigorous results indicated by the solid lines, as a function of evolution time (in atomic units) for both the subprocesses modeling the many-body diffusion process of two three-dimensional identical fermions and three-dimensional free-distinguishable-particle diffusion. 

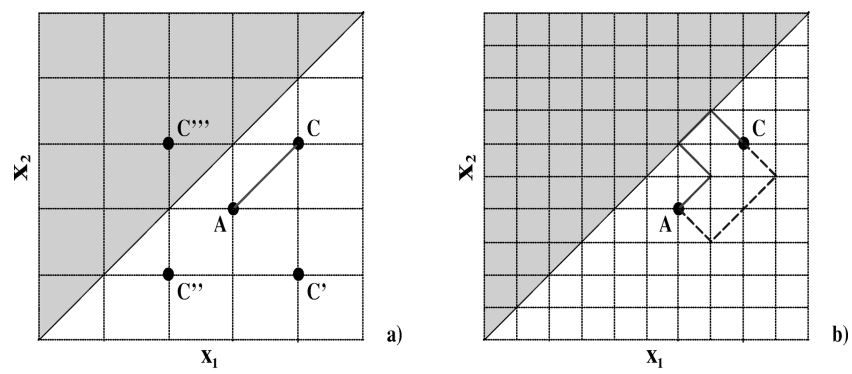

FIG. 6. The definition of finite time steps and corresponding spatial grids induces shortcomings with respect to the accurate assignment of validity flags. The refinement of the coarse grid in (a) indicates [see (b)] that a considerable rate of possible paths from $A$ to $C$ enters prohibited (gray) regions.

processes $\{f, b, b\},\{b, f, b\}$, and $\{b, b, f\}$. As can be clearly seen in Fig. 5, the processes decay with a different rate; the reason for this is that the distribution of the first-passage time $\tau_{\partial D}$ depends on the initial position. For large times, $\tau \rightarrow \infty$, they decay as $\tau^{-1 / 2}$ proportional to the relative fermionlike coordinate of the initial position. Again, considering the state space as the domain of interest, the evolution of the exit time for distinguishable particles reveals a totally different decay in time, because nothing prevents them from reentering the domain after having left it before.

\section{SAMPLING THE GROUND STATE}

The sample paths inside the domain are made using the traditional techniques for Brownian motion or Bernoulli walks. In addition, computational efficiency calls for the incorporation of the Feynman-Kac functional using the technique of branching and killing. This is consistent with the underlying formalism of many-body diffusion provided the system does not require walker transitions from an $\ell$ state to a (different) $\ell^{\prime}$ state, thereby changing its boundary conditions. The fact that such transitions are possible in a general potential has been overlooked in [3] (see [16]). However, it remains of interest to simulate a class of antisymmetric states sign-problem-free with the proposed algorithm; in particular, in view of algorithms for more advanced models taking into account the additional transitions from $\ell$ to $\ell^{\prime}$.

\section{A. Estimation of the first-passage time}

Although the consequences of permutation symmetry are rigorously incorporated in both the formalism of many-body diffusion and the algorithm described so far, numerical practice reveals that the simulation of the imaginary-time Schrödinger equation along these lines with discrete time steps introduces systematic inaccuracies due to the possibility of crossing and recrossing the boundary during that finite time step. To elucidate this point, we focus on the evolution of free particles, for the effect is caused by the underlying kinetics, the potential here being irrelevant. Consider two identical fermions on a line, with their coordinates in the configuration space denoted as $x_{1}$ and $x_{2}$ (see Fig. 6). The absorbing boundary is given by

$$
\partial D_{2}=\left\{x_{1}=x_{2}\right\} .
$$

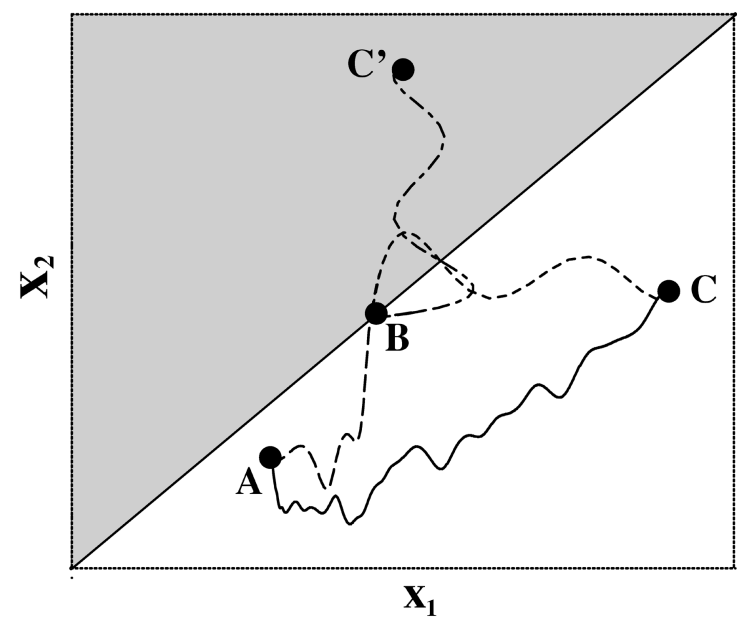

FIG. 7. Diffusers having propagated from $A$ to $C$ may have followed a valid path (solid line) as well as an invalid one (dashed line $A B C$ ) that passed into the forbidden region (in gray) outside the domain.

Assume that a discrete time scale $\Delta \tau$ and, consistently according to the Einstein relation, a spatial grid with spacings $\Delta x=\sqrt{\Delta \tau}$ has been chosen, as illustrated by Fig. 6(a). During a time step $\Delta \tau$, a walker initially located at $A$ could move a distance $\Delta x$ into each direction, so as to reach one of the points $C, C^{\prime}, C^{\prime \prime}$, or $C^{\prime \prime \prime}$. Let it eventually advance to $C$. The only possibility to perform that step on the broad grid is thus the straight move, as indicated by the straight solid line $A C$ in Fig. 6(a). Refinement of the grid [see Fig. 6(b)] to spacings $\Delta x / 2$, and correspondingly time steps $\Delta \tau / 4$, indicates that not all possible paths from $A$ to $C$ provide nonzero contributions. During the four time steps $\Delta \tau / 4$, the diffuser either could have taken admissible paths $A C$ like the dashed one or, on the other hand, could meanwhile have left the domain as indicated by the solid path $A C$. In the latter case, the move would be invalid and the diffuser should be absorbed. But the broad grid in Fig. 6(a) principally does not allow us to distinguish between valid and invalid paths $A C$. In other words, working on any (artificial) discrete grid, any move the initial and final positions of which are situated inside the domain automatically includes a set of invalid paths. This gives rise to systematic errors in the evolution.

\section{B. The crossing-recrossing correction}

To correct for these errors in the evolution, each trial displacement of a diffuser must be assigned a ratio of validity, which directly corresponds to the ratio of possible valid and invalid paths to perform the move in continuous space. The correction has to do with the crossing and recrossing at the boundary in a time lapse smaller than the time increment used in the simulation. It may be illustrated by the following arguments.

A probabilistic argument based on the reflection principle can be given as follows. Suppose that a walker, situated at $A$, evolves to $C$ (see Fig. 7). It might either have taken a completely valid path (solid line) or have hit the boundary at, e.g., $B$ (dashed line $A B C$ in Fig. 7). Because free distinguishable particle diffusion is isotropic, the probability $P\left[X_{\tau+\Delta \tau}=C \mid X_{\tau}=A, X_{\nu}=B\right]$ to reach $C$ over an arbitrary 
point $B$ on $\partial D_{2}$ with $\nu \in[\tau, \tau+\Delta \tau]$ equals the probability $P\left[X_{\tau+\Delta \tau}=C^{\prime} \mid X_{\tau}=A, X_{v}=B\right]$ to end up in the image $C^{\prime}$. Clearly, the total probability for the move from $A$ to $C$ is the sum of the two conditional probabilities to move either from $A$ to $C$ without crossing $B$ or to move from $A$ to $C^{\prime}$ with crossing $B$. Generalizing this argument to all points $B$ on the boundary, the move from $A$ to $C$ has to take into account two nonintersecting subsets of paths, i.e.,

$$
\begin{aligned}
P\left[X_{\tau+\Delta \tau}=C \mid X_{\tau}=A\right]= & P_{\partial}\left[X_{\tau+\Delta \tau}=C \mid X_{\tau}=A\right] \\
& +P_{\widehat{\partial}}\left[X_{\tau+\Delta \tau}=C \mid X_{\tau}=A\right],
\end{aligned}
$$

where the index $\partial$ means that the path crosses the boundary, while $\widetilde{\partial}$ indicates that $C$ is reached without boundary crossing-recrossing. The (conditional) probability $P_{\partial}\left[X_{\tau+\Delta \tau}\right.$ $\left.=C \mid X_{\tau}=A\right]$ equals $P_{\partial}\left[X_{\tau+\Delta \tau}=C^{\prime} \mid X_{\tau}=A\right]$, and since any move from $A$ to $C^{\prime}$ crosses the boundary one obtains

$$
\begin{aligned}
P\left[X_{\tau+\Delta \tau}=C \mid X_{\tau}=A\right]= & P\left[X_{\tau+\Delta \tau}=C^{\prime} \mid X_{\tau}=A\right] \\
& +P_{\bar{\partial}}\left[X_{\tau+\Delta \tau}=C \mid X_{\tau}=A\right] .
\end{aligned}
$$

The crossing-recrossing correction can then be identified with

$$
\begin{aligned}
P_{\partial}\left[X_{\tau+\Delta \tau}=C \mid X_{\tau}=A\right]= & P\left[X_{\tau+\Delta \tau}=C \mid X_{\tau}=A\right] \\
& \times\left(1-\frac{P\left[X_{\tau+\Delta \tau}=C^{\prime} \mid X_{\tau}=A\right]}{P\left[X_{\tau+\Delta \tau}=C \mid X_{\tau}=A\right]}\right) .
\end{aligned}
$$

Hence, the normalized probability for moves from $A$ to $C$ with boundary crossing-recrossing arises from the ratio

$$
\begin{aligned}
R(A \rightarrow C)= & \frac{P\left[X_{\tau+\Delta \tau}=C^{\prime} \mid X_{\tau}=A\right]}{P\left[X_{\tau+\Delta \tau}=C \mid X_{\tau}=A\right]} \\
& =\exp \left\{-\frac{\left(x_{1}^{\prime}-x_{2}^{\prime}\right)\left(x_{1}-x_{2}\right)}{\Delta \tau}\right\} .
\end{aligned}
$$

A physical argument leading to the same correction is based on the method of the images implying that the evolution of two walkers without interaction but with absorbing boundary conditions is given by the determinant of the free propagators. Factoring out the diagonal element one obtains the same expression. In practice, the evolution procedure must be extended with the supplementary rejection ratio (9): even diffusers having made a move to a final position which is clearly situated inside the domain may eventually be judged invalid.

As the many-body diffusion formalism traces back the evolution of many-body systems of arbitrary spatial dimension to adapted one-dimensional diffusion processes, the argument described above is readily generalized to any dimensions. The extension to $N$ particles with, e.g., ordering along the $x$ axis corresponds to the construction of $N-1$ significant boundary conditions. The probability to cross the set of boundaries during the move from $A$ to $C$, both points ele- ments of the domain, is complementary to the probability not to hit any of the $N-1$ boundaries. Hence, a completely valid path occurs according to the product of elemental probabilities of the form $1-R(A \rightarrow C)$. The implementation of these extended probabilities eliminates the systematic errors originating from finite-time-step considerations in the presence of absorbing boundaries. Strictly speaking, the systematic errors specific for our numerical description of many-fermion systems compares with systematic inaccuracy attributing to the simulation of respective distinguishable-particle systems.

It must be stressed that the arguments concerning boundary crossing-recrossing effects are reminiscent of the method of image charges, which have been transferred to probability theory a long time ago [18]. The method is based on the mathematical formalism of diffusion as distinct from other up-to-date quantum Monte Carlo techniques. Quite in contrast, the same geometrical argument has been employed by Anderson [19] to formulate the behavior at boundaries fixed by the nodal structure of a trial function and by [20] to introduce the so-called Pauli potential.

\section{ESTIMATION OF GROUND-STATE ENERGIES}

Most often propagator-based methods will be used to estimate the energy of the system under consideration. In this case, one can use the exponential decay of the free energy, or one may devise separate estimators for the components of the internal energy. Propagator-based methods use implicitly paths with known beginning and ending points. The key path integral goes over all paths starting and ending in the same point of the phase space. In the algorithm explained in the preceding section only a construction of sample paths with a known starting point is given. A generalization of the construction, keeping the increments independent and imposing an end point but taking the boundary into account, is to our knowledge not documented in the mathematical literature. Without the boundary condition the construction is known as the Brownian bridge; physically it means that one takes the classical action as the starting point to incorporate quantum fluctuations. Without the generalization of the Brownian bridge another estimation scheme for the ground-state energy has to be used.

\section{A. The kinetic contribution}

To the best of our knowledge, it was Anderson [21] who first reported ground-state-energy estimates based on the $a$ priori assumption of the exponential ground-state-energy dependence of the time decay of the propagator. For distinguishable-particle systems, the ground-state energy can be evaluated by "stochastic" averages over the potential $V(\bar{r})$,

$$
E_{0}=\lim _{\tau \rightarrow \infty} E(\tau)=\lim _{\tau \rightarrow \infty} \frac{\int d \bar{r} \Psi(\bar{r}, \tau) V(\bar{r})}{\int d \bar{r} \Psi(\bar{r}, \tau)}=\langle V\rangle^{s} .
$$

The "stochastic estimates" $E(\tau)$ are based on the stochastic probability density 


$$
\Psi(\bar{r}, \tau)=\mathbb{E}_{R}\left[\exp \left(-\int_{0}^{\tau} V(R(\tilde{\tau})) d \tilde{\tau}\right)\right]
$$

For sufficiently large Euclidean time $\tau, \Psi(\bar{r}, \tau)$ mimics the ground-state wave function and, accordingly, the estimates $E(\tau)$ reflect the ground-state energy $E_{0}$.

The expectation in Eq. (11) goes over all sample paths starting at $\bar{r}$ and ending wherever in the configuration space with $\{X(\tau) ; \tau \geqslant 0\}$ a Brownian motion or a random walk. This type of functional integration leading to a wave function is based on the path-integral representation of the wave operator [22]. Once a procedure for the wave function is found, it is obvious that the potential average (10) is in direct contrast to the quantum-mechanical expectation value of the potential, and both potential averages must be clearly distinguished.

Antisymmetry, as specific for the ground-state wave function of identical fermions, causes the breakdown of the potential average (10) as an estimate of the ground-state energy in the configuration space. Both the numerator and the denominator in the estimator tend to zero: the projection on the antisymmetric representation of the simulated wave function in the asymptotic limit of large Euclidean time $\tau$ is not well defined anymore. During the thermalization of the system, the statistical error attached to the numerical estimates rapidly overwhelms the signal.

Replacing the Brownian motion or random walk over the configuration space by the many-body diffusion process as discussed in the preceding section, the formulation of energy estimates adapted to the state space $D_{N}$ with its boundary conditions avoids the mentioned decay of the signal-to-noise ratio. The implementation of the many-body diffusion process goes hand in hand with a loss of walkers at the absorbing boundaries of the state space. From the algorithmic point of view, this loss is caused by the underlying diffusion kinetics and contains essential information on the lowest energy of that antisymmetric state. In terms of the wave function on the domain, Eq. (10) has to be replaced by

$$
\begin{array}{r}
E_{0}=\lim _{\tau \rightarrow \infty} E_{\tau}=-\frac{\left(\hbar^{2} / 2 m\right) \int_{\partial D_{N}} d \bar{r} \bar{\nabla} \Psi_{F}(\bar{r})}{\int_{D_{N}} d \bar{r} \Psi_{F}(\bar{r})} \\
+\frac{\int_{D_{N}} d \bar{r} \Psi_{F}(\bar{r}) V(\bar{r})}{\int_{D_{N}} d \bar{r} \Psi_{F}(\bar{r})}=\langle j\rangle^{s}+\langle V\rangle^{s},
\end{array}
$$

where $\Psi_{F}(\bar{r})=\Psi_{F}(\bar{r}, \infty)$ denotes the asymptotic probability density on the state space $D_{N}$ with the boundaries $\partial D_{N}$ :

$$
\Psi_{F}(\bar{r}, \tau)=\mathbb{E}_{R_{F}}\left[\exp \left(-\int_{0}^{\tau} V\left(R_{F}(\tilde{\tau})\right) d \tilde{\tau}\right)\right],
$$

where $\left\{X_{F}(\tau) ; \tau \geqslant 0\right\}$ is a many-body diffusion or many-body random walk.

In contrast to the distinguishable-particle estimator (10), Eq. (12) comprises additional kinetic contributions $\langle j\rangle^{s}$. In
TABLE I. Comparison of eigenenergies $E$ (a.u.) gained by the many-body diffusion algorithm, with results from rigorous analytical calculations.

\begin{tabular}{cccccccccc}
\hline \hline$\Omega$ & $\omega$ & $N$ & $N_{\uparrow}$ & $N_{\downarrow}$ & $D$ & $E$ & $\sigma$ & $E_{\text {exact }}$ & $E_{\text {exc }}$ \\
\hline 2 & 1 & 2 & 2 & 0 & 1 & 3.1215 & 0.0010 & 3.1213 & 1.4142 \\
2 & 1 & 2 & 2 & 0 & 3 & 6.5356 & 0.0013 & 6.5355 & 1.4142 \\
2 & 1 & 3 & 3 & 0 & 3 & 9.0091 & 0.0051 & 9.0000 & 3.0000 \\
3 & 1 & 4 & 2 & 2 & 3 & 19.039 & 0.0074 & 19.034 & 4.4721 \\
3 & 1 & 6 & 2 & 4 & 3 & 29.594 & 0.0185 & 29.615 & 12.124 \\
3 & 1 & 8 & 7 & 1 & 3 & 35.991 & 0.0209 & 36.000 & 21.000 \\
4 & 1 & 10 & 10 & 0 & 3 & 149.27 & 0.0989 & 149.30 & 110.23 \\
4 & 1 & 10 & 5 & 5 & 3 & 88.118 & 0.0472 & 88.058 & 48.990 \\
5 & 1 & 20 & 10 & 10 & 3 & 272.52 & 0.2795 & 272.47 & 201.25 \\
\hline \hline
\end{tabular}

the case of fermions, $\langle j\rangle^{s}$ can be interpreted as the relative flow per time step through the absorbing boundaries of the state space; this expectation value depends on the distribution of the first-passage time $\tau_{\partial D}$. In the standard treatment of distinguishable particles, $\bar{\nabla} \Psi(\bar{r})$ at the corresponding (infinitely remote) boundaries is zero. Evidence that the gradient of diffusive movement at reflecting boundaries vanishes has been given in [3]. For a diffusion or a random walk of the $\{f, b, b\}$ type, for example, the kinetics reveals nonzero gradients at the absorbing boundaries in the $x$ direction. Consequently, surface terms $\langle j\rangle^{s}$ are of essential importance in our treatment.

\section{B. The model system}

The algorithm introduced in the preceding section has been used to calculate the lowest energy of the states specified by a given symmetry for an oscillator model with the following Hamiltonian:

$$
H=-\sum_{j=1}^{N} \frac{\hbar^{2}}{2 m} \Delta_{j}+\sum_{j=1}^{N} \frac{m \Omega^{2}}{2} \vec{r}_{j}^{2}-\sum_{i, j=1}^{N} \frac{m \omega^{2}}{2}\left(\vec{r}_{i}-\vec{r}_{j}\right)^{2} .
$$

The repulsive interparticle interaction is introduced to mimic many-body interaction. Because Eq. (14) can be diagonalized, all the energies of the system are known analytically. The diagonalization, the thermodynamic properties, and the response properties of this model have been discussed elsewhere $[13,14]$. The ground states of Eq. (14) for identical particles with a specific symmetry (i.e., specifying the boundary conditions on the domain $D_{N}^{3}$ ) are excited states of Eq. (14), considered as a system of distinguishable particles. The purpose of showing that the algorithm is workable has led us to choose the worst cases: the particles are arranged in such a way that the ranking according to all their coordinates is maintained during the simulation, and fermion diffusion is chosen along one direction, while boson diffusion is chosen for the other directions. The simulation has been done for several values of $\Omega$ and $\omega$ indicated in Table I. $N=N_{\uparrow}$ $+N_{\downarrow}$ indicates the number of particles in the system. The $N_{\uparrow}$ spin-up particles and the $N_{\downarrow}$ spin-down particles are mutually indistinguishable, but the $N_{\uparrow}$ spin-up particles are distinguishable from the $N_{\downarrow}$ spin-down particles. $D$ is the spatial dimension of the evolution equation for each particle. $E$ is 


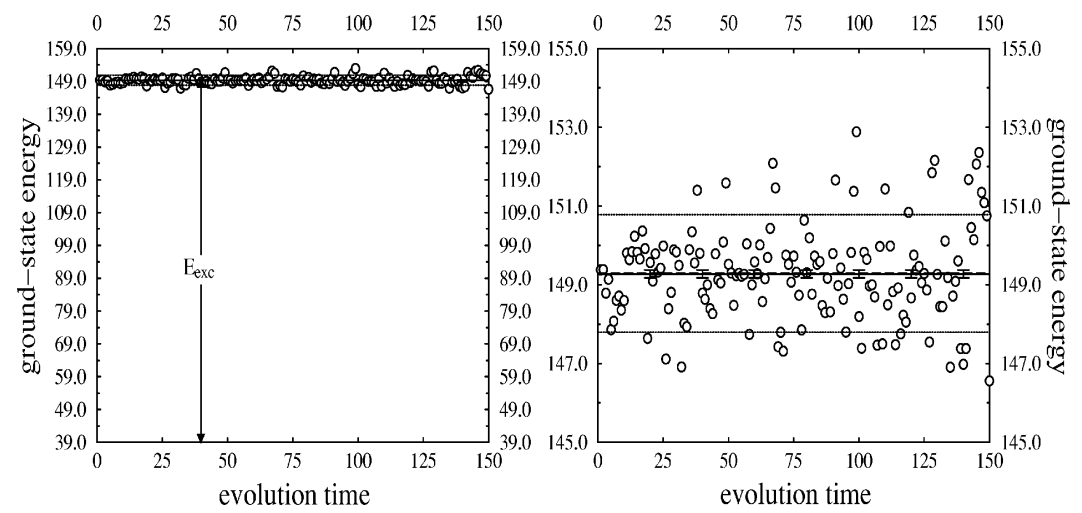

FIG. 8. Ground-state-energy estimates in atomic units (circles) for ten three-dimensional identical fermions, Eq. $(14), \Omega=4, \omega=1$ as a function of evolution time in atomic units on two different scales. The dotted lines at the right-hand side indicate a deviation of $1 \%$ from the rigorous ground-state energy (solid line). The numerically predicted energy is denoted by the dashed line. The left-hand side illustrates the same results on the scale of exchange contributions.

the energy obtained from the simulation, $\sigma$ is the standard deviation, and $E_{\text {exact }}$ is the analytically obtained energy value of the excited state of the distinguishable oscillator model corresponding with the symmetry as explained above. In order to have an idea about the relevance of the flux term and of the boundary conditions, the exchange energy $E_{\text {exc }}$ is the difference between $E$ and the ground-state energy of the system of distinguishable particles. Time step periods of $10^{-3}$ atomic units proved suitable for the parameter values listed in Table I. In Fig. 8 the numerical results for ten threedimensional identical fermions are shown.

The present results confirm the accurate implementation of quantum statistics for the model of indistinguishable interacting harmonic oscillators. In all the considered cases, the many-body diffusion algorithm has achieved energy estimates, which are in good agreement with the analytical rigorous results. We stress that our results do not significantly depend on the choice of the initial densities. In particular, the support of sophisticated trial functions has been avoided in the present paper, for the emphasis is on the investigation of the algorithm's general capability to describe indistinguishable particles.

\section{DISCUSSION AND CONCLUSIONS}

It should be emphasized that in all the simulations that we have reported here, use has been made of the special symmetry property of the model under study. Its Hamiltonian is invariant under permutations of the Cartesian components of the coordinates. For motion in three dimensions, this invariance allows us to define four independent partial processes, each with a well-defined boundary condition. Introducing a number of walkers, initially randomly divided over the four types of processes, their absorption determines how many walkers survive. The absorption together with the reflection at the boundary $\partial D_{N}^{3}$ determines the distribution in $D_{N}^{3}$. This has been illustrated for a few low-dimensional examples. Given that the distribution is understood, an estimator for the ground-state energy is obtained. This estimator takes the outflow of the walkers out of the domain into account as a crucial ingredient in the ground-state energy. In order to study the statistical errors two common simulation methods have been considered; both the inclusion of Bernoulli walks or diffusion leads to analogous results if a suitable time step is chosen (that also determines the unit of distance by the fundamental diffusion constant $\hbar / 2 m$ through the Einstein relation) and if the crossing-recrossing corrections for absorbing boundaries are applied. The ground-state-energy estimation is satisfactory, especially taking into consideration that these estimates are obtained without invoking importance sampling. Furthermore, the estimation is sign-problemfree.

A discussion of the present results is not an easy matter when placed against the large number of publications partially dealing with this problem. In the long run, it will be the ability to treat realistic models with an extension of this method that will determine its usefulness. Without further discussion and without being exhaustive the following references deal with such models: [9-11,23].

In summary, in this paper a sign-problem-free simulation of a specific interacting fermion system is presented. The simulated system has harmonic interactions, and the algorithm as well as the theory leading to this algorithm make explicit use of the harmonic character of the interactions. Apart from this limitation, it is shown that the algorithm based on the absorption and reflection at a boundary of a Brownian motion or a Bernoulli walk reproduces the correct distributions. It gives also good energy estimates when crossing and recrossing correction on the first-exit time are taken into account. In view of the fact that no importance sampling has been used an acceptable standard deviation has been obtained.

\section{ACKNOWLEDGMENTS}

This work was supported by the Interuniversity Poles of Attraction Program, Belgian State, Prime Minister's Office, Federal Office for Scientific, Technical and Cultural Affaires. We acknowledge support from the BOF NOI 1997 projects of the University of Antwerpen, NFWO Project No. WO.073.94N (Wetenschappelijke Onderzoeksgemeenschap, 
Scientific Research Community of the NFWO on "LowDimensional Systems', ), and the European Community Program Human Capital Mobility through Contract No. CHRXCT93-0124. Part of this work has been performed in the framework of Project No. G.0287.95 and the supercomputer
Project No. 1.5.729.94N of the FWO. F.L. is very grateful to B. Gerlach for partial financial support during the development of this work. F.B. acknowledges the Fonds voor Wetenschappelijk Onderzoek-Vlaanderen for financial support.
[1] L.F. Lemmens, F. Brosens, and J.T. Devreese, Phys. Lett. A 189, 473 (1994).

[2] F. Brosens, J.T. Devreese, and L.F. Lemmens, Solid State Commun. 96, 137 (1995).

[3] L.F. Lemmens, F. Brosens, and J.T. Devreese, Phys. Rev. E 53, 4467 (1996).

[4] W.M.C. Foulkes, Phys. Rev. Lett. 71, 2158 (1993); M. Caffarel, D.M. Ceperley, and M.H. Kalos ibid. 71, 2159 (1993).

[5] A. Korzeniowski, J.L. Fry, D.B. Orr, and N.G. Fazleev, Phys. Rev. Lett. 69, 893 (1992); 71, 2160 (1993).

[6] K.E. Schmidt and M.H. Kalos, in Applications of the Monte Carlo Method in Statistical Physics, edited by K. Binder (Springer, Berlin, 1984).

[7] D.M. Ceperley and B.J. Alder, Phys. Rev. Lett. 45, 566 (1980); Physica B \& C 108B, 875 (1981); J. Chem. Phys. 81, 5833 (1984); J.W. Moskowitz, K.E. Schmidt, M.A. Lee, and M.H. Kalos, ibid. 77, 349 (1982); P.J. Reynolds, D.M. Ceperley, B.J. Alder, and W.A. Lester, Jr., ibid. 77, 5593 (1982).

[8] M. Caffarel and P. Clavarie, J. Chem. Phys. 88, 1088 (1988); 88, 1100 (1988).

[9] M. Takahashi and M. Imada, J. Phys. Soc. Jpn. 53, 963 (1984).

[10] W.H. Newman and A. Kuki, J. Chem. Phys. 96, 1409 (1992).
[11] R.W. Hall, J. Chem. Phys. 97, 6481 (1992).

[12] D.A. Butts and D.S. Rokshar, Phys. Rev. A 55, 4346 (1997).

[13] F. Brosens, J.T. Devreese, and L.F. Lemmens, Phys. Rev. E 55, 227 (1997).

[14] F. Brosens, J.T. Devreese, and L.F. Lemmens, Phys. Rev. E 55, 6795 (1997).

[15] F. Brosens, J.T. Devreese, and L.F. Lemmens, Phys. Rev. A 55, 2453 (1997).

[16] L.F. Lemmens, F. Brosens, and J.T. Devreese, Phys. Rev. E 55, 7813 (1997).

[17] R.N. Bhattacharya and E.C. Waymire, Stochastic Processes with Applications (J. Wiley and Sons, New York, 1990).

[18] W. Feller, An Introduction to Probability Theory and its Applications, 3rd ed. (Wiley, New York, 1968).

[19] J.B. Anderson, J. Chem. Phys. 65, 4121 (1976).

[20] O.O. Tursunov and O.V. Zhirov, Phys. Rev. Lett. 222, 110 (1989).

[21] J.B. Anderson, J. Chem. Phys. 63, 1499 (1975).

[22] G. Roepstorff, Path Integral Approach To Quantum Mechanics (Springer, Berlin, 1994).

[23] C. Chakravarty, J. Chem. Phys. 99, 8038 (1993). 